\title{
Psicopatología de las compulsiones de lavado en el trastorno obsesivo- compulsivo: No todos los pacientes lavan por los mismos motivos
}

\author{
Gemma García-Soriano ${ }^{1}$, Carmen Carrió ${ }^{2}$ y Amparo Belloch ${ }^{1}$ \\ ${ }^{1}$ Universidad de Valencia, Valencia, España \\ ${ }^{2}$ Hospital Arnau de Vilanova-Lliria, Valencia, España
}

\begin{abstract}
Resumen: El trastorno obsesivo-compulsivo (TOC) es un trastorno heterogéneo en cuanto al contenido de las obsesiones y compulsiones. El lavado, una de las compulsiones más frecuentes, se ha asociado a diferentes variables como: el miedo al contagio físico o mental, el miedo al afecto negativo y a la pérdida de control, un elevado perfeccionismo o la evitación de la sensación de suciedad o de inacabado. En este trabajo se examinan estas variables a través del análisis descriptivo de la sintomatología psicopatológica y la respuesta al tratamiento de tres pacientes con diagnóstico principal de TOC que realizan rituales de lavado. Tanto el análisis clínico como la evaluación a través de autoinformes muestran la heterogeneidad motivacional que subyace a una misma expresión sintomática, el lavado, y sugieren la necesidad de tener esta variable en cuenta en el abordaje terapéutico individualizado de cada paciente.
\end{abstract}

Palabras clave: TOC; compulsiones; lavado; dimensiones de síntomas; contaminación.

Psychopathology of washing compulsions in obsessive-compulsive disorder: Not all patients wash for the same reasons

\begin{abstract}
Obsessive-compulsive disorder (OCD) is a heterogeneous disorder in terms of the contents of obsessions and compulsions. Washing, being one of the most frequent compulsion modalities, has been associated with different variables such as: physical or mental contamination fears, fears associated with negative affect (including disgust) and the loss of control, a high level of perfectionism, or the avoidance of a sense of dirtiness or incompleteness. In this study, these variables are examined through the descriptive analysis of the psychopathological symptoms and treatment responses of three patients with OCD and washing rituals as their main diagnosis. Both the clinical analysis and the evaluation through self-reports reflect the motivational heterogeneity associated with a same symptom expression, washing, and suggest the need to consider these variables in the individualized treatment of each patient.
\end{abstract}

Keywords: OCD; compulsions; washing; symptom dimensions; contamination.

\section{Introducción}

El trastorno obsesivo-compulsivo (TOC), caracterizado por la presencia de obsesiones y/o compulsiones

Recibido: 21 de enero 2016; aceptado: 25 febrero 2016.

Correspondencia: Gemma García-Soriano, Facultad de Psicología, Universidad de Valencia, Avda. Blasco Ibáñez 21, 46010, Valencia, España. Correo-e: gemma.garcia@uv.es

Agradecimientos: Este trabajo forma parte de los proyectos de investigación PSI2013-44733-R financiado por el Ministerio de Economía y Competitividad (Gobierno de España), y PROMETEO-2013/066, financiado por la Conselleria d' Educació, Cultura i Esport (Generalitat Valenciana, Valencia). clínicamente significativas, se describe como un trastorno homogéneo por los actuales sistemas de diagnóstico (DSM-5, American Psychiatric Association [APA], 2013; ICD-10, World Health Organization [WHO], 1992). Sin embargo, la heterogeneidad de los contenidos obsesivos (p. ej., contenidos sexuales, blasfemos, de contaminación u orden) es bien conocida y su estudio está recibiendo una importante atención en la literatura científica en parte debido a las posibles implicaciones que parece tener la diversidad sintomatológica en la respuesta terapéutica (p.ej., Abramowitz, Franklin, Schwartz, y Furr, 2003). Se estima que un $25.7 \%$ de las personas con TOC presentan preocupaciones relaciona- 
das con la contaminación (Ruscio, Stein, Chiu, y Kessler, 2010), y que, entre aquellos pacientes que solicitan ayuda, un $37.80 \%$ presenta obsesiones de contaminación y un $26.50 \%$ compulsiones de limpieza (Foa et al., 1995).

Habitualmente, cuando se describe a los pacientes con síntomas de contaminación, no se distingue entre "tipos" de síntomas (o de pacientes). Sin embargo, en la literatura se han identificado diferentes fuentes de amenaza percibidas por los pacientes con TOC que recurren al lavado compulsivo (García-Soriano, Belloch, y Mori1lo, 2008). La definición misma de qué es un contaminante o qué implica contagio, difiere enormemente entre los pacientes: mientras que para unos pacientes está claramente definido e implica contacto con contaminantes físicos (p.ej., virus, bacterias, veneno), para otros los contaminantes pueden estar relacionados con pensamientos o ideas sin necesidad de que medie el contacto físico, por tanto, temiendo la sensación misma de sentirse contaminado (o sucio) (Tolin y Meunier, 2007). En este sentido, Rachman (1994) ha descrito que las compulsiones de lavado pueden ser la respuesta: (a) a una sensación de suciedad física; (b) al miedo a desarrollar una enfermedad como consecuencia de la contaminación; o (c) a una sensación de suciedad "interna" o de contaminación mental, que puede ser provocada por pensamientos, palabras o recuerdos, y por tanto, sin necesidad de contacto físico. El miedo sería la emoción predominante entre quienes temen la enfermedad o contaminarse (b, c), mientras que el malestar y el asco podrían ser también característicos de quienes lavan porque se sienten sucios (a). Esta propuesta ha sido ampliada por Tallis (1996b) al sugerir que hay pacientes que, sin estar preocupados por la posibilidad de contaminarse, lavan (d) debido a un elevado perfeccionismo y a la necesidad de "preservar la percepción de que los objetos valiosos están en un perfecto estado" (p. 362), o para alcanzar un estado de limpieza perfecta.

Feinstein, Fallon, Petkova, y Liebowitz (2003), a partir de una serie de análisis factoriales del listado de síntomas del Yale-Brown Obsessive-Compulsive Scale (Goodman et al., 1989a; Goodman et al., 1989b), propusieron que la sintomatología asociada a la contaminación se agrupaba en dos factores diferentes. Un primer factor que incluía ítems referidos a la preocupación o al asco asociados a las secreciones corporales, la suciedad y/o los gérmenes, y donde predominaba el malestar; y otro factor asociado tanto al miedo a enfermar como a transmitir algún tipo de enfermedad, y donde predominaba el miedo al daño. Estas últimas obsesiones se agrupaban en un mismo factor con las obsesiones de contenido violento o agresivo (hacia uno mismo o hacia otros) —dado que ambas implican miedo, y por tanto, evitación del daño-y con los rituales de comprobación. La presencia de estos dos factores en relación a la contaminación se ajusta a la propuesta respecto al TOC de Rasmussen y Eisen (1991), desarrollada más recientemente por el grupo de Summerfeldt (p.ej., Summerfeldt, Kloosterman, Antony, y Swinson, 2014; Summerfeldt, 2004). Estos autores plantean que en el TOC subyacen dos grandes dimensiones motivacionales: la de inacabado y la de evitación del daño. La sensación de inacabado se ha definido como un estado interno de imperfección o experiencia de que las cosas no están como deberían de estar que se asocia a una motivación y estado afectivo diferentes a los relacionados con la ansiedad, y que se experimentaría como malestar, insatisfacción o tensión (Summerfeldt, 2004). Un concepto cercano al de inacabado, es el de "experiencias no del todo correctas" (not just right experiences, NJRE) que hace referencia a la aparición, asociada con determinados estímulos, de una sensación incómoda de que hay algo que no está del todo bien, de que no se ha alcanzado el estado deseado de perfección (Belloch et al., 2016; Carrasco y Belloch, 2013; Coles, Frost, Heimberg, y Rhéaume, 2003; Fornés-Romero, Ruiz, y Belloch, 2016). Tanto la sensación de inacabado como las experiencias no del todo correctas se han asociado al perfeccionismo (Coles et al., 2003; Wahl, Salkovskis, y Cotter, 2008) y, por lo tanto, podrían estar relacionadas con la propuesta de Tallis (1996) de que hay pacientes cuyo lavado compulsivo se debe a la necesidad de alcanzar un estado de limpieza "perfecto". La segunda dimensión motivacional, la evitación del daño, se asocia al miedo, y podría estar en la base de la motivación que lleva a algunos pacientes a lavarse porque temen enfermar o contagiar/-se, tal y como describe Rachman (1994).

Cougle et al. (2007) también han diferenciado entre dos motivaciones subyacentes al lavado compulsivo: (a) el miedo a un estado afectivo negativo (incluido el asco) y a la sensación de pérdida de control generada por la suciedad, y (b) la necesidad de evitar la enfermedad. Otros autores proponen que las variables motivacionales subyacentes al miedo a la contaminación serían la evitación del asco y la evitación del daño (Melli, Chiorri, Carraresi, Stopani, y Bulli, 2015). En relación al papel que juega el asco, cabe destacar que tanto la propensión como la sensibilidad al asco, no sólo son relevantes en los trastornos de ansiedad (Sandín, Chorot, Santed, Valiente, y Olmedo, 2008; Sandín, Valiente, Chorot, Santed y Pineda, 2013) sino también en el TOC con síntomas de contaminación (p.ej., Arnáez, García-Soriano, y Belloch, 2015; Goetz, Lee, Cougle, y Turkel, 2013; Sandín, Chorot, Valiente, y Santed, 2014). 
Teniendo en cuenta estas aproximaciones, parece que la mayor parte de los autores están de acuerdo en que una parte de los pacientes con compulsiones de lavado están motivados a lavar por la tendencia a evitar el daño físico o mental (evitación de la enfermedad o contagio); otros autores describen a pacientes motivados por la sensación de suciedad; el afecto negativo (incluido el asco) y pérdida de control asociado a la contaminación; o la evitación de la sensación de inacabado o de experiencias "not just right". Estas variables motivacionales pueden conceptualizarse como dimensiones no opuestas que pueden darse a la vez en los mismos pacientes (Rachman, 1994)

La existencia de diferentes motivaciones subyacentes a los rituales de lavado podría tener implicaciones terapéuticas, y ser una de las variables que expliquen las diferencias en la respuesta al tratamiento entre los pacientes con obsesiones de contaminación y compulsiones de lavado. En este sentido, se ha observado que los pacientes que tienen claramente definidas las consecuencias de no realizar los rituales (p.ej., enfermar o contagiar a alguien) responden mejor al tratamiento de exposición que aquellos pacientes en los que los temores no están tan delimitados (Foa, Abramowitz, Franklin, y Kozak, 1999), como es el caso de pacientes que lavan motivados por la evitación del asco o por las sensaciones de inacabado. En esta misma línea, Cougle et al. (2007) han informado de mejores respuestas al tratamiento de exposición con prevención de respuesta entre aquellos que presentaban un elevado miedo a la enfermedad versus quienes no presentaban ese temor. Estos autores sugieren que los resultados pueden explicarse por la emoción subyacente a cada variable motivacional (miedo versus asco), y la respuesta diferencial de ambas emociones a la exposición, que es más lenta y atenuada en el caso del asco. Por lo tanto, parece importante tener en cuenta el contenido de las obsesiones y compulsiones predominantes en los pacientes con TOC a la hora de desarrollar tratamientos específicos, ya que, incluso dentro de un mismo subtipo o dimensión (p.ej., la contaminación), pueden ser relevantes diferentes variables (p.ej., miedo a enfermar o miedo a la sensación de suciedad) para explicar síntomas idénticos (p.ej., lavado). Por tanto, es muy probable que se necesiten enfoques terapéuticos diferentes para abordar esta heterogeneidad (Belloch et al., 2014; Sookman, Abramowitz, Calamari, Wilhelm, y McKay, 2005).

El objetivo de este trabajo es estudiar la heterogeneidad sintomática de las compulsiones de lavado en el TOC. Para ello nos centraremos en el análisis de tres casos clínicos, atendiendo especialmente a la presentación de síntomas, a la formulación clínica de los casos, a la gravedad del TOC, a la respuesta al tratamiento, y a las variables emocionales, cognitivas y motivacionales implicadas.

\section{Método}

\section{Participantes}

Paciente 1. Mujer de 36 años de edad, soltera y con estudios superiores. Está desempleada desde hace un año. Vive con sus padres. Tiene una hermana mayor casada y con un hijo.

Paciente 2. Hombre de 28 años de edad, soltero y con estudios de formación profesional. Actualmente trabaja como operario de una fábrica. Vive con su padre. Tiene dos hermanos mayores casados. Hace 6 años que mantiene una relación sentimental con una mujer de su edad.

Paciente 3. Hombre de 45 años de edad, casado y con estudios superiores. Está empleado en el sector servicios. Vive con su esposa. No tiene hijos. Tiene dos hermanos.

Los 3 casos fueron atendidos en una unidad de salud mental comunitaria del sistema sanitario público español. A todos ellos les atendió en primer lugar un psiquiatra y éste fue quien los derivó a la psicóloga clínica. La evaluación por parte de la psicóloga clínica se realizó entre diciembre de 2013 (pacientes 1 y 2) y enero de 2014 (paciente 3). En todos los casos el tratamiento psicológico se inició un mes después.

\section{Evaluación}

La evaluación previa al tratamiento de cada paciente se realizó de forma individualizada a lo largo de tres sesiones en las que el clínico realizó una entrevista diagnóstica y completó el Y-BOCS (Yale-Brown Obsessive-Compulsive Scale-Severity; Goodman et al., 1989a; Goodman et al., 1989b. Versión española de Nicolini et al., 1996). Los pacientes completaron una serie de instrumentos de autoinforme con la ayuda del clínico en el caso de que fuera necesaria, y también se llevó a cabo el análisis funcional del problema. Una vez finalizado el tratamiento, el clínico volvió a valorar la gravedad de los síntomas obsesivo-compulsivos a través de la Y-BOCS, y los pacientes completaron el Inventario de Creencias Obsesivas Revisado (Belloch et al., 2010). Esta evaluación se realizó de nuevo al cabo de un año de haber finalizado el tratamiento.

Entrevista clínica. En primer lugar, se empleó una entrevista semiestructurada que permitió recoger datos 
demográficos, así como la información necesaria para completar la historia clínica y evaluar las conductas problema objeto del tratamiento. A continuación, se empleó el ADIS-IV (Anxiety Disorders Interview Schedule for DSM-IV: Life-time versión; Di Nardo, Brown, y Barlow, 1994) para confirmar el diagnóstico clínico de trastorno obsesivo-compulsivo, y valorar la presencia de otros trastornos comórbidos. Todos los pacientes presentaron un diagnóstico principal de TOC de acuerdo con los criterios diagnósticos del DSM-IV-TR (APA, 2000) y del DSM-5 (APA, 2013).

Valoración de la gravedad. Como ya se ha comentado, se empleó la escala de gravedad Y-BOCS. El Paciente 1 presentaba un TOC muy grave, el paciente 2 grave, y el paciente 3 moderado (ver Tabla 1).

Cuestionario de experiencias no del todo correctas revisado (NJRE-Q, Not Just Right Experiences Questionnaire-Revised; Coles, Frost, Heimberg, y Rhéaume, 2003; traducción al castellano de Carrasco y Belloch, 2013). Se trata de un cuestionario de autoinforme con 19 ítems que consta de tres partes. En la primera se valora la presencia en el último mes de 10 experiencias de inacabado (NJRE-frecuencia). En la segunda se le pide al paciente que indique la experiencia de este tipo experimentada más recientemente, y cuándo se produjo. A continuación se valora esa experiencia en las siguientes 7 dimensiones desde 1 ("nada") hasta 7 ("extremadamente"): frecuencia, intensidad, molestia a corto plazo, molestia a largo plazo, rumiación, necesidad de responder, y grado de responsabilidad asociado a la experiencia. La puntuación combinada de las 7 dimensiones se considera un indicador de gravedad de la experiencia "not just right" seleccionada. Este instrumento ha mostrado una consistencia interna de entre 0.63 (primera parte) y 0.85 (tercera parte) en muestras españolas (Carrasco y Belloch, 2013). El paciente 1 informó de 7 experiencias "not just right" en el último mes, frente a 2 y 5 en los pacientes 2 y 3 respectivamente. Los pacientes 1 y 2 seleccionan la misma experiencia "not just right" como la más reciente: "Después de lavarme las manos una vez, he tenido la sensación de que no las sentía como se supone que se sienten las manos limpias" e informaron haberla experimentado en las últimas horas, mientras que el paciente 3 seleccionó la experiencia "Después de vestirme, he tenido la sensación de que mi ropa (etiquetas, cuellos, perneras de los pantalones, etc.,) me molestaba", experimentada por última vez durante la última semana. Al valorar estas experiencias "not just right", el paciente 1 presentó una mayor gravedad que los otros dos pacientes (ver Figura 1).

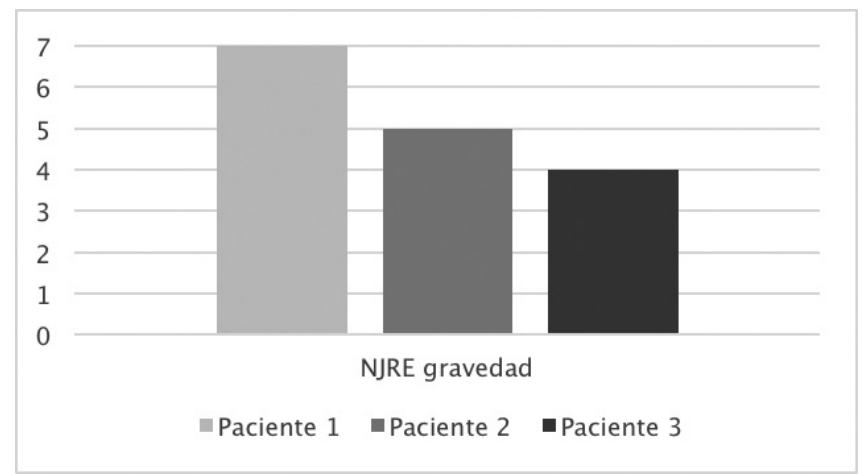

Figura 1. Comparación de las puntuaciones obtenidas por los pacientes en el Cuestionario de experiencias no del todo correctas revisado (NJRE-Q).

Cuestionario de dimensiones nucleares obsesivo-compulsivas (OC-CDQ; Obsessive-Compulsive Core Dimensions Questionnaire; Summerfeldt et al., 2014; versión en castellano: Carrasco y Belloch, 2013). Está formado por 20 ítems que evalúan (desde 1 "nunca se aplica a mi" hasta 5 "siempre se aplica a mi") la evitación del daño, y la sensación de inacabado. La consistencia interna es elevada para ambas escalas (0.89 y 0.88 , respectivamente) (Summerfeldt et al., 2014). Para cada escala se ha obtenido la puntuación media. El paciente 1 presentó mayor puntuación en sensación de inacabado y menor en evitación del daño. Los pacientes 2 y 3 presentaron puntuaciones similares en las dos escalas, siendo más elevadas las del paciente 2 (ver Figura 2).

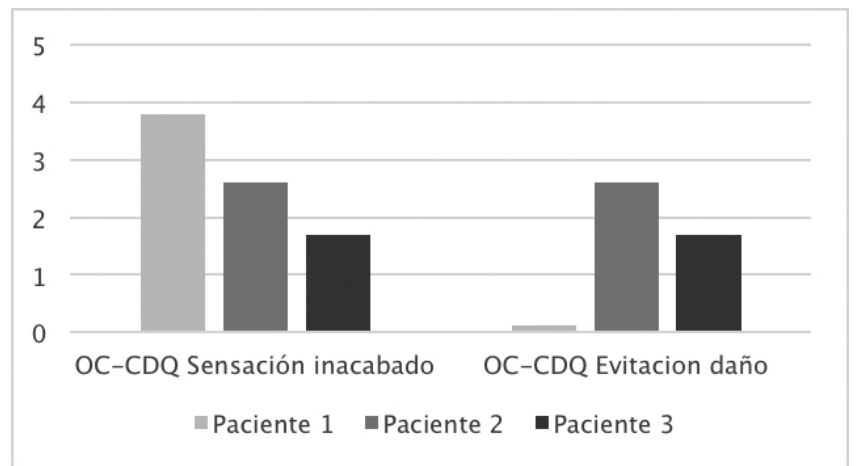

Figura 2. Comparación de las puntuaciones obtenidas por los pacientes en el Cuestionario de dimensiones nucleares obsesivo-compulsivas (OC-CDQ).

Escala de propensión y sensibilidad al asco (DPSS-R; Disgust Propensity and Sensitivity Scale-Revised; van Overveld, de Jong, Peters, Cavanagh, y Davey, 2006; adaptación española de Sandín, Chorot, Olmedo, y Valiente, 2008). Consta de 16 ítems valorados desde 1 "nunca" hasta 5 siempre", que se agrupan en dos esca- 
las: propensión al asco o tendencia a experimentar asco, y sensibilidad al asco o sensación de desagrado ante las experiencias de asco. Estas escalas han mostrado una buena consistencia interna ( 0.76 y 0.73 respectivamente) (Sandín et al., 2008). El paciente 1 presentó menores puntuaciones en las escalas de asco que los otros dos pacientes. El paciente 2 presentó la mayor tendencia a experimentar asco (propensión) y el paciente 3 la mayor sensibilidad al asco (ver Figura 3).

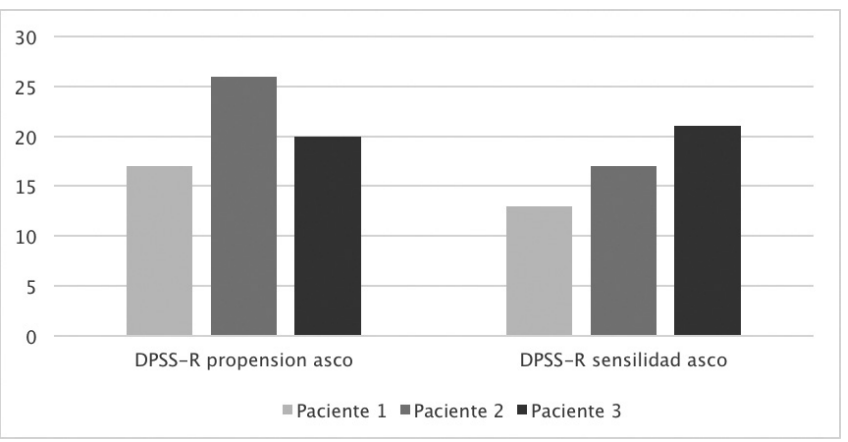

Figura 3. Comparación de las puntuaciones obtenidas por los tres pacientes en cada subescala de la Escala de propensión y sensibilidad al asco (DPSS-R).

Inventario de Creencias Obsesivas Revisado (ICO-R; Belloch et al., 2010) incluye 50 ítems que se estiman desde 1 ("totalmente en desacuerdo") hasta 7 ("totalmente de acuerdo"), y se agrupan en ocho escalas de creencias disfuncionales sobre los pensamientos: responsabilidad excesiva; importancia de los pensamientos; fusión pensamiento acción (FPA) tipo probabilidad; FPA tipo moral; importancia de controlar los pensamientos; sobreestimación del peligro; intolerancia a la incertidumbre; y perfeccionismo. La consistencia interna de estas escalas se sitúa entre 0.69 (responsabilidad excesiva) y 0.85 (perfeccionismo) (Belloch et al., 2010). La puntuación de cada escala se ha calculado obteniendo la puntuación media en la escala. En la evaluación pre-tratamiento los tres pacientes presentaron una elevada puntuación en las creencias de responsabilidad y en la importancia de controlar los pensamientos. El paciente 1 presentó además un elevado perfeccionismo y el paciente 2 una elevada intolerancia a la incertidumbre. En la Tabla 1 se recogen las puntuaciones antes y después del tratamiento así como en el seguimiento a un año.

\section{Motivo de consulta y formulación clínica}

A continuación se describe el motivo de consulta y la formulación clínica de los tres pacientes según el modelo cognitivo del TOC (p.ej., Salkovskis, 1999).

\section{Paciente 1}

Solicita ayuda debido a la interferencia que le produce el problema en su vida: imposibilidad de independizarse porque se ve incapaz "de poder limpiar, arreglar, decorar,... mi piso"; no viaja porque no se considera capaz de hacer la maleta "nunca consigo tener la sensación de que ya está bien hecha, como toca,... ”; discusio-

Tabla 1. Puntuaciones en los instrumentos Y-BOCS e ICO-R en tres momentos diferentes: pre-tratamiento, post-tratamiento y seguimiento a un año

\begin{tabular}{|c|c|c|c|c|c|c|c|c|c|}
\hline & \multicolumn{3}{|c|}{ Paciente 1} & \multicolumn{3}{|c|}{ Paciente 2} & \multicolumn{3}{|c|}{ Paciente 3} \\
\hline & Pre & Post & 1 año & Pre & Post & 1 año & Pre & Post & 1 año \\
\hline Y-BOCS total & 34 & 12 & 11 & 28 & 8 & 8 & 18 & 6 & 7 \\
\hline Y-BOCS obsesiones & 18 & 6 & 6 & 14 & 4 & 4 & 9 & 3 & 2 \\
\hline Y-BOCS compulsiones & 16 & 6 & 5 & 14 & 4 & 4 & 9 & 3 & 5 \\
\hline ICO-R Responsabilidad & 3.8 & 3.2 & 3.1 & 4.2 & 3 & 3.4 & 4 & 3.4 & 3.1 \\
\hline ICO-R Importancia pensamientos & 1.6 & 1.2 & 1.2 & 1 & 1 & 1 & 2.4 & 2.6 & 2.8 \\
\hline ICO-R FPA-probabilidad & 1.2 & 1 & 1 & 1 & 1 & 1 & 3.2 & 3.6 & 2.8 \\
\hline ICO-R FPA-moral & 2.2 & 2.2 & 2.1 & 2.2 & 3 & 1 & 3.7 & 2.8 & 2.4 \\
\hline ICO-R Importancia controlar los pensamientos & 5.4 & 3.4 & 3.2 & 5.4 & 1 & 3.4 & 4 & 3.4 & 2.5 \\
\hline ICO-R Sobrestimación del peligro & 3.8 & 3.3 & 3 & 4.3 & 2.2 & 2.8 & 3.6 & 3.7 & 2.9 \\
\hline ICO-R Intolerancia a la incertidumbre & 4.3 & 3.6 & 3.5 & 5.8 & 3.2 & 3.7 & 2.8 & 3 & 2.5 \\
\hline ICO-R Perfeccionismo & 5.7 & 4 & 3.9 & 3.8 & 2.3 & 2 & 2.4 & 2.6 & 2 \\
\hline
\end{tabular}

Nota . FPA = fusión pensamiento-acción; ICO-R = Inventario de creencias obsesivas revisado; Y-BOCS = Escala de gravedad de síntomas obsesivo-compulsivos de Yale-Brown 
nes y enfrentamientos crecientes con sus padres debido a su sintomatología: "me molesta que toquen mis cosas, ... no soporto que me las cambien de sitio,... y menos que dejen algo mío en el suelo" (p.ej., que desplacen su bolso del sofá al suelo), "mis padres no respetan mis manías", etc. La paciente es consciente de la irracionalidad de sus "manías", del coste temporal que le suponen y del grado de interferencia en su funcionamiento ("esto no es vida"). Presenta un TOC de 18 años de evolución, y rasgos de personalidad obsesivo-compulsiva.

Se han identificado una serie de variables que pueden haber facilitado el desarrollo del problema, su mantenimiento o recurrencia. Entre los mismos cabe destacar el estilo educativo sobreprotector presentado especialmente por la madre, y las características de personalidad de la paciente, que ella misma define como de alta autoexigencia y perfeccionismo, así como "rígida" y "obstina$d a "$.

En el momento de la evaluación, el cuadro obsesivo-compulsivo viene caracterizado por los siguientes síntomas: en primer lugar, obsesiones relacionadas con: (a) la limpieza y contaminación que se concretan en la sensación de asco o suciedad cuando toca cualquier cosa, siendo necesario tener continuamente las manos limpias (en palabras de la paciente "no notar nada en ellas"; es decir, tener la sensación de que están limpias, sin restos de suciedad). Manifiesta no tener miedo a la contaminación ni al contagio, pero necesita tener la sensación de tenerlas limpias permanentemente, de "notar que no tengan nada"; (b) la necesidad de que su arreglo personal (maquillaje, manicura, depilación cejas, limpieza de su cuerpo y cabello,...) quede perfecto; (c) la necesidad de que su dormitorio esté limpio, impoluto; y (d) la necesidad imperiosa de no percibir ninguna imperfección en las prendas de vestir que se compra (hilos sueltos, defectos en la confección del tejido, señales de las etiquetas o cabellos ...)

Estas obsesiones aparecen ante las siguientes situaciones (factores precipitantes): (a) tocar cualquier cosa en su casa, el polvo que se desprende al limpiar; (b) en el momento de aseo y arreglo personal; (c) al ordenar o limpiar su dormitorio, y (d) al adquirir nuevas prendas de vestir y al vestirse con ropa nueva.

Para disminuir la molestia generada por las obsesiones y por no tener las manos "limpias", la paciente emplea una serie de estrategias de neutralización. Entre las compulsiones, emplea con mayor frecuencia, en primer lugar, el lavado de manos inmediatamente después de tocar cualquier objeto con el objetivo de que estén "como toca" (esta compulsión se da tras las situaciones a y c). Estos lavados han llegado a provocar el sangrado de las manos. En segundo lugar (a partir de la situación b), el arreglo personal ritualizado con numerosas comprobaciones de que todo queda "bien", "esté perfectamente limpio", dedicando un número excesivo de horas (p.ej., 3 horas para hacerse la manicura o para ducharse). En tercer lugar, a partir de la situación c, la limpieza ritualizada de su habitación de modo que cada vez que toca un objeto que pudiera tener polvo se lava las manos antes de tocar el siguiente objeto. Por último (a partir de la situación d), comprobaciones generales de que las cosas están como tienen que estar y de que las prendas de ropa no tienen imperfecciones. Estas estrategias, provocan a corto plazo un alivio del malestar generado por las obsesiones, pero facilitan la permanencia de las valoraciones disfuncionales asociadas a las mismas, y, por tanto, mantienen el malestar generado por las mismas y su aparición a largo plazo. Además, para evitar la aparición de sus obsesiones y el malestar asociado, evita diferentes acciones que "sé que podrían desencadenar mis obsesiones". Entre las mismas destaca: (en relación a la situación b) evita dedicarse ella al aseo y/o arreglo personal de diversas maneras: o no lo realiza (p.ej., no se hace la manicura o la depilación de cejas), o va a un salón de belleza para que se lo hagan, y le pide a su madre que le lave el cabello, o demora todo lo que puede ducharse; (en relación a la situación c) delega la limpieza de su habitación a su madre. El hecho de que la madre limpie la habitación de la paciente, a corto plazo hace que la paciente se sienta mejor y no tenga que implicarse en una labor que le llevaría muchas horas, a la vez que refuerza la conducta de evitación de la paciente y facilita el mantenimiento del problema. Además (en relación a la situación d), evita utilizar la ropa nueva que adquiere y la almacena en su armario sin llegar a sacarla de la bolsa de la tienda donde la ha comprado. De este modo, evita involucrarse en el proceso de comprobar que no existen imperfecciones en la ropa. Esta conducta tiene como consecuencia la acumulación de prendas y calzado en su armario. Como puede apreciarse, la paciente utiliza fundamentalmente como estrategias de neutralización los rituales de lavado y la evitación activa y pasiva.

\section{Paciente 2}

El paciente solicita ayuda porque desde hace 7 meses presenta intrusiones sobre dudas que ha tratado de evitar sin éxito, que le generan gran malestar, y a las que tiene que dedicar mucho tiempo.

Se han identificado una serie de acontecimientos estresantes durante el año anterior al inicio del TOC que pudieron actuar como factores precipitantes o desencadenantes remotos: (1) la enfermedad y muerte súbita de la madre del paciente. A raíz de ello, el paciente comien- 
za a realizar atribuciones de responsabilidad-culpa y sobrevaloración del peligro (p.ej., "la tenía que haber tratado de forma diferente", "no reñirla ni enfadarme con ella cuando no se acordaba de lo que le habia dicho", "no le presté la atención que se merecía", etc.); (2) los cambios en la fábrica donde trabajaba que suponen una fuente de estrés al ampliar su horario laboral (11 horas/ día); (3) el visionado de un programa de televisión sobre enfermedades de transmisión sexual que tuvo un elevado impacto emocional para el paciente. Además, se describe como una persona responsable, meticulosa, aprensiva y sugestionable, variables que pudieron actuar como factores predisponentes.

El cuadro obsesivo-compulsivo se caracteriza por la presencia de obsesiones de: (a) contaminación, concretamente temor a contagiarse de alguna enfermedad y trasmitirla a las personas de su entorno, y (b) dudas y necesidad de comprobar posibles errores o descuidos al realizar cualquier tarea cotidiana. Las obsesiones relacionadas con la contaminación (a) aparecen precipitadas por una serie de variables como ver una mancha roja en la máquina del trabajo (p.ej., de tinta o suciedad), en la calle o en su casa; ver algún material del botiquín en el trabajo o material sanitario en la calle (p.ej., jeringuilla); tocar algún objeto que haya tocado alguien que tenga una herida en las manos, o que pueda estar sucio. Las obsesiones asociadas a la necesidad de comprobación (b) suelen aparecer cuando el paciente tiene que cerrar el grifo después de cepillarse los dientes, cerrar la puerta de la casa a la vuelta del trabajo, apagar los fuegos de la cocina, poner el despertador, cerrar el coche o apagar la máquina del trabajo.

Para hacer frente a la ansiedad generada por las obsesiones y para evitar aquello que teme (contagio de una enfermedad y cometer algún error con consecuencias desastrosas), realiza las siguientes estrategias de control que le sirven para atenuar el malestar y disminuir el miedo a la contaminación y a los errores, pero que actúan como variables mantenedoras del problema. Realiza (a) compulsiones de lavado de manos (con agua y jabón, y toallitas húmedas) que le llegan a irritar las manos, así como lavado de objetos tocados por personas que pudieran tener heridas en las manos. Además, evita una serie de acciones con el objetivo de evitar el contagio, como las siguientes: que la ropa y el calzado del trabajo estén en contacto con sus otras prendas de vestir y calzado; tocar objetos que puedan ocasionar algún contagio (los coge utilizando toallitas húmedas); emplear guantes en el trabajo para manipular la maquinaria. También realiza (b) compulsiones de comprobación consistentes en comprobar entre 7 y 8 veces seguidas que ha cerrado el gas, la puerta, etc.

\section{Paciente 3}

Acude a consulta animado por su esposa quien, desde hace 10 años nota ciertas manías fuera de lo común, pero que el paciente minimiza, refiriendo únicamente alguna queja sobre la presencia de pensamientos de contenido agresivo y el tiempo empleado en realizar determinados rituales de lavado. Se identifican como variables que han podido facilitar la aparición de algunos de los síntomas, la presencia en su entorno más cercano de conductas de acumulación de objetos por parte de su madre y tío materno "ellos lo reutilizaban y reparaban todo" (aprendizaje vicario, desencadenantes remotos).

Presenta diferentes obsesiones relacionadas con: (a) la necesidad de lavarse o limpiarse de una manera determinada y un determinado número de veces tanto las manos, la cara, como los dientes y los genitales, para sentir la sensación de que está suficientemente limpio; (b) dudas sobre si ha realizado algunas acciones (p.ej., cerrado el grifo); (c) dudas sobre si en un futuro próximo va a necesitar determinados objetos; y (d) el impulso de salirse del carril mientras conduce en la autovía y provocar así un accidente.

Estas obsesiones se desencadenan cuando: (a) va a llevar a cabo el aseo personal sobre todo a la hora de lavarse los dientes, cara y genitales; (b) sale de casa, del coche, etc.; (c) ve un objeto que pudiera ser útil en el futuro (folios en la papelera, tornillos, clavos, o piezas metálicas); y (d) conduce por la autovía.

Para contrarrestar el malestar generado por sus obsesiones, realiza una serie de comportamientos (estrategias de control) que le sirven para aliviar el malestar provocado por sus obsesiones, pero que a su vez, mantienen el problema. En primer lugar, presenta compulsiones de (a) lavado de manos, cara, dientes y genitales que realiza un número concreto de veces y en un orden determinado; (b) de comprobación, consistentes en comprobar dos o tres veces que ha cerrado las puertas de casa, del coche, apagado el gas, las luces, etc.; (c) acumulación, que le llevan a almacenar en botes y cajas aquellos objetos que piensa que pueden ser reutilizados en un futuro. Además, para hacer frente a su miedo a provocar un accidente (d) evita conducir por la autovía. También emplea estrategias de distracción cognitiva poniéndose música cuando tiene las obsesiones.

\section{Intervención}

Siguiendo las pautas de tratamiento descritas en Belloch, Cabedo y Carrió (2011), se emplearon dos módulos terapéuticos: (1) terapia cognitiva específica (TCE) y (2) terapia de exposición con prevención de respuesta 
(E+PR). Además, se comenzó con sesiones de psicoeducación en las que se introdujo el modelo explicativo cognitivo-conductual del TOC.

\section{Paciente 1}

Con esta paciente se llevaron a cabo 2 sesiones de psicoeducación sobre el modelo explicativo cognitivo-conductual del TOC y se decidió aplicar el módulo de tratamiento de E+PR dirigido a disminuir o eliminar las conductas de neutralización (compulsiones, evitación, conductas para reasegurarse,...) dado que éstas actúan como reforzadores para la aparición de pensamientos obsesivos, incrementando su frecuencia. Dicha decisión se tomó por las siguientes razones: a) la rigidez cognitiva de la paciente iba a dificultar un abordaje cognitivo inicial, y b) el uso por parte de la paciente, además de los rituales de lavado, de la evitación activa y pasiva como estrategia de neutralización, y el efecto negativo que ésta tenía sobre su calidad de vida. Además, también se trabajó la acomodación familiar, se incidió en que los padres y su hermana cambiaran las conductas que estaban reforzando el problema, ya que se consideró que era otro de los factores básicos de mantenimiento del problema y del deterioro de las relaciones intrafamiliares. En total, se llevaron a cabo 18 sesiones de E+PR ( 5 se realizaron en el domicilio de la paciente) y 2 sesiones de prevención de recaídas. Además, se llevaron a cabo 8 sesiones familiares. Esta paciente presentaba además un estado de ánimo depresivo y, antes de iniciar el tratamiento psicológico, ya estaba bajo tratamiento psiquiátrico-farmacológico (Fluoxetina $60 \mathrm{mgr} . / \mathrm{dí}$; Aripiprazol $5 \mathrm{mgr}$./ día; Bupropión $5 \mathrm{mgr}$./día). En el momento de finalización de la intervención psicológica se mantenía la misma medicación.

\section{Paciente 2}

Después de realizar 3 sesiones de psicoeducación sobre el modelo explicativo cognitivo-conductual del TOC, se aplicó únicamente el módulo de TCE. Dicho módulo terapéutico se centra en la modificación o reestructuración de las creencias disfuncionales sobre los pensamientos y las valoraciones erróneas básicas que el paciente hace de sus obsesiones. En este paciente las creencias disfuncionales que se abordaron fueron: la sobreestimación de la importancia del pensamiento, la fusión pensamiento-acción tipo probabilidad (i.e., la creencia de que solo por el mero hecho de pensar en algo aumenta la probabilidad de que ocurra lo pensado), la responsabilidad excesiva, la importancia de controlar el pensamiento, la sobrevaloración del peligro y la intole- rancia a la incertidumbre. El número total de sesiones fue de 14, seguidas de otras 2 de prevención de recaídas. El paciente, antes de iniciar el tratamiento psicológico, ya estaba sometido a un tratamiento psicofarmacológico pautado por su psiquiatra (Escitalopram $20 \mathrm{mgr}$./día), y al finalizar la intervención psicológica ya no tomaba ningún tipo de medicación.

\section{Paciente 3}

Tras 3 sesiones psicoeducativas centradas en el modelo explicativo cognitivo-conductual del TOC, se aplicaron los dos módulos de tratamiento TCE y EP+R. En primer lugar, se aplicó la TCE durante un total de 8 sesiones, en las que se trabajaron las siguientes creencias disfuncionales: la fusión pensamiento-acción tipo probabilidad y moral, la responsabilidad excesiva, la importancia de controlar el pensamiento, y la sobrevaloración del peligro. En segundo lugar se aplicó el módulo de $\mathrm{E}+\mathrm{PR}$ a lo largo de 12 sesiones y al término del mismo se realizaron 2 sesiones de prevención de recaídas. Este paciente voluntariamente decidió seguir únicamente tratamiento psicológico, por lo que no tomó ninguna medicación.

En la Tabla 1 se ofrecen los datos referentes a la gravedad (Y-BOCS) y puntuación media del nivel de creencias obsesivas (ICO-R) previas al tratamiento, después del tratamiento, y en el seguimiento de un año. Se observa una reducción clínicamente significativa de los niveles de gravedad en todos los pacientes, así como una reducción de las creencias disfuncionales. Estos niveles se mantuvieron en el seguimiento de un año.

\section{Discusión}

Los tres pacientes descritos presentaban un diagnóstico principal de TOC con síntomas de contaminación, y compulsiones de lavado (de manos, dientes y genitales, y de objetos), así como evitación de situaciones que pudieran "facilitar" la contaminación o la necesidad de implicarse en el lavado. Además, todos los pacientes manifestaban otros contenidos de obsesiones y compulsiones (p.ej., de comprobación).

Centrándonos en la sintomatología que nos ocupa, la de lavado, observamos que las obsesiones y el patrón de lavado diferían entre los tres pacientes. En el caso del paciente 1, el lavado de manos y la limpieza de la habitación se producían por la necesidad de que las cosas estuvieran como tienen que estar y por hacer frente a una sensación de malestar que surgía si las cosas no estaban de un modo determinado. Esta información, obtenida a través de la entrevista, se ve apoyada por la información 
recogida a través de los autoinformes, que reflejaban una elevada frecuencia de experiencias "not just right", así como una alta gravedad asociada a la mismas, junto con una elevada experimentación de sensaciones de inacabado, mayor que la informada en otros trabajos con muestras de pacientes con TOC (Belloch et al., 2016; Ecker, Kupfer, y Gönner, 2014; Ghisi, Chiri, Marchetti, Sanavio, y Sica, 2010). Cabe destacar que el ítem seleccionado como experiencia "not just right" más reciente se corresponde con el lavado de manos. Además, se observa una muy baja tendencia a la evitación del daño (puntuaciones cercanas al 0 ), apoyando la idea de que la paciente no teme la contaminación o contagio de una enfermedad. Destacan sus elevadas creencias disfuncionales sobre el perfeccionismo (medidas a través del instrumento ICO-R), que apoyan su auto-descripción y sus rasgos de personalidad obsesivo-compulsiva descritos por la terapeuta. Como se ha indicado previamente, las asociaciones entre las experiencias de inacabado y el perfeccionismo han sido apuntadas previamente en la literatura (Wahl et al., 2008). Este paciente es el que obtuvo las puntuaciones más bajas en sensibilidad y propensión al asco, si bien fueron algo más elevadas que las informadas en la literatura como típicas de los pacientes con TOC (Olatunji, Tart, Ciesielski, McGrath, y Smits, 2011). Los síntomas de esta paciente se ajustarían a la descripción de Tallis (1996) de aquellos pacientes que lavan motivados por la necesidad de alcanzar un estado de perfección. Esta necesidad de perfección se observa también en otros de sus rituales como las comprobaciones repetidas de que las prendas compradas no tienen imperfecciones, o las tareas de aseo personal como la manicura, que han de están perfectamente realizadas. Así mismo, se ajustaría a las descripciones de quienes han diferenciado entre el lavado motivado por la sensación de inacabado versus el motivado por la evitación del daño (Cougle et al., 2007). Esta paciente mostraba también el TOC de mayor gravedad y duración. Este factor, junto con sus rasgos de personalidad motivaron el comenzar el tratamiento por el módulo de E+PR. No se consideró necesario añadir el módulo de TCE.

En el caso del paciente 2, observamos que la motivación subyacente al lavado de manos y de objetos (p.ej., maquinaria) se asocia al miedo a transmitir o contraer alguna enfermedad por contagio. Este caso es el que mejor se ajusta a la definición clásica de las obsesiones de contaminación. El paciente tiene claramente identificado el modo de transmisión del contagio: la sangre; miedo que extiende a cualquier mancha u objeto que pueda recordarle a la sangre. Presenta, además, las mayores puntuaciones en la escala de evitación del daño, lo cual es congruente con la idea de que estos pacientes están motivados por evitar un posible daño hacia sí mismos o los demás. La puntuación en esta escala es elevada en comparación con los otros dos pacientes, y con otras muestras de pacientes con TOC (Belloch et al., 2016). Respecto a la dimensión de "not just right", también selecciona la experiencia de "not just right" asociada al lavado de manos, que le genera una molestia menor que a la paciente 1 , aunque sigue siendo elevada y mayor a la media de los pacientes con TOC (Belloch et al., 2016; Ghisi et al., 2010). La sensación de inacabado es moderada en comparación con los otros pacientes, y similar a la informada en trabajos que incluyen muestras de pacientes con TOC (Belloch et al., 2016; Ecker et al., 2014). Este paciente informa de una mayor tendencia a experimentar asco (propensión al asco) ante diferentes situaciones que los otros pacientes, siendo esta tendencia mayor a la descrita en la literatura en pacientes con TOC (Olatunji et al., 2011). Además, presenta mayor sensibilidad al asco que la media de pacientes con TOC (Olatunji et al., 2011). También muestra una elevada intolerancia a la incertidumbre, tal vez vinculada al miedo a enfermar, y a la imposibilidad de tener la certeza de que la contaminación temida no se ha producido. Los datos procedentes de la entrevista y de los auto-informes, son congruentes con la descripción del paciente como muy responsable y meticuloso, así como con la descripción realizada en la literatura de los pacientes que lavan movidos por el miedo a contraer o contagiar una enfermedad, y por tanto, motivados por la evitación del daño. Aunque se ha planteado en la literatura que en los pacientes que temen la enfermedad la emoción predominante sería la ansiedad (y no el asco) (Cougle et al., 2007; Rachman, 1994), en el caso de este paciente, el asco también parece jugar un papel relevante. Respecto a la respuesta al tratamiento, únicamente se aplicó un módulo de terapia cognitiva, no siendo necesario aplicar $\mathrm{EP}+\mathrm{R}$. Este dato resulta interesante puesto que se ha planteado que los pacientes que tienen identificada la amenaza responderían mejor al tratamiento de exposición (Foa et al., 1999).

El paciente 3 presentaba un lavado-limpieza ritualizados de manos, cara, dientes y genitales que debía realizar en un orden determinado y un número de veces concreto. En este paciente, la motivación subyacente al lavado, según la evaluación a través de la entrevista, se asociaba a la necesidad de sentir que las cosas están limpias. Si examinamos sus puntuaciones en los instrumentos de auto-informe observamos que presentó niveles de tendencia a las experiencias de inacabado y de evitación del daño similares e inferiores a los informados por pacientes con TOC (Belloch et al., 2016; Ecker et al., 2014). Las experiencias de "not just right" también pre- 
sentaron una "gravedad" algo inferior a la media informada en muestras con pacientes heterogéneos de TOC (Belloch et al., 2016; Ghisi et al., 2010). Respecto a la sensibilidad y propensión al asco, manifestó puntuaciones moderadas y similares en cuanto a la tendencia a experimentar asco, y en cuanto a responder con angustia ante la emoción de asco, por lo que su necesidad de lavado podría estar asociada también con estas variables. Los valores en esta escala (sensibilidad y propensión al asco) son mayores a los informados en la literatura respecto a los pacientes con TOC (Olatunji et al., 2011). El paciente había establecido unos criterios particulares para alcanzar ese estado de limpieza a través de un número de repeticiones y el orden en que se produce el lavado. Este criterio, de elevado contenido mágico, se ve apoyado por sus elevadas puntuaciones en las creencias de fusión pensamiento acción tipo probabilidad y moral. Es el paciente que presenta las puntuaciones más elevadas en esas creencias, es decir, en la idea de que por pensar algo es más fácil que suceda, o que pensar algo es equivalente a hacerlo. También es el paciente que presenta una conciencia de enfermedad menor. Es probable que, precisamente por ello, valore que sus comportamientos de evitación le interfieren poco y perciba que les dedica poco tiempo. A partir de ahí, resulta asimismo comprensible que sus puntuaciones en el Y-BOCS sean menores que las de los otros pacientes, ya que este instrumento es una entrevista estructurada que completa el clínico sobre la base de la información verbal que aporta el paciente. En cierto modo, sería un ejemplo de "lavador" sin miedo a la enfermedad y donde la evitación del daño no sería la variable motivacional más relevante. $\mathrm{Su}$ patrón de lavado se ajustaría a los pacientes descritos por Rachman (1994) que lavan porque temen la sensación de suciedad. En estos pacientes no está tan delimitada la emoción predominante, y el asco, como sucede en este caso, podría jugar un papel relevante. Precisamente fue en este paciente, en el que la amenaza no estaba tan claramente establecida como en el anterior, donde hubo que aplicar los dos módulos de tratamiento, y requirió un mayor número de sesiones $(\mathrm{n}=25)$ pese a su menor gravedad inicial, evaluada a través del Y-BOCS.

Pese a las limitaciones asociadas a un estudio descriptivo como este y centrado únicamente en tres casos clínicos, los resultados a partir de la presentación de tres pacientes con diagnóstico de TOC y sintomatología predominante asociada al lavado de manos, apoyan una serie de aspectos tratados en la literatura científica sobre el TOC que pueden tener importantes implicaciones a la hora de conceptualizar y tratar el trastorno. En primer lugar, los datos clínicos apoyan la heterogeneidad de los síntomas de lavado en el TOC, a través de la presencia de al menos tres tipos de "lavadores": quienes lavan por la necesidad de perfección, quienes lo hacen para evitar la enfermedad, y quienes lavan para eliminar la suciedad. Estas descripciones se ajustarían a las de Rachamn (1994) y Tallis (1996).

En segundo lugar, este trabajo apoya la relevancia que tienen en la contaminación tanto las variables cognitivas, como las variables motivacionales y emocionales. Respecto a las variables cognitivas, nuestros datos sugieren que las creencias de sobreestimación del peligro, responsabilidad e importancia de los pensamientos, son relevantes en la contaminación (Obsessive Compulsive Cognitions Working Group, 2005; Tolin, Brady, y Hannan, 2008). Además, hay creencias que parecen ser más relevantes en unas modalidades de contaminación que en otras, diferencias que podrían explicar las inconsistencias entre estudios a la hora de determinar qué creencias son más relevantes para qué síntomas OC (García-Soriano y Belloch, 2013; Smith, Wetterneck, Hart, Short, y Björgvinsson, 2012; Viar, Bilsky, Armstrong, y Olatunji, 2011). Respecto a las variables emocionales, nuestros datos avalan la relevancia para los síntomas de contaminación de las dos dimensiones de asco (Arnáez et al., 2015; García-Soriano, Rose11-Clari, y Serrano, 2015; Sandín et al., 2014), cuya importancia parece diferir entre pacientes. Por lo que se refiere a las variables motivacionales, nuestros datos apoyan la relevancia de estas dimensiones de forma diferencial para las diferentes modalidades de contaminación (Cougle, Goetz, Fitch, y Hawkins, 2011), y contrastan con aquellos trabajos que han sugerido que la sensación de inacabado no se relaciona con las compulsiones de lavado, sino con otras manifestaciones del TOC (Chik, Calamari, Rector, y Riemann, 2010; Ecker y Gönner, 2008).

En tercer lugar, este trabajo sugiere que las variables relevantes para cada modalidad de contaminación no son independientes, y que, al menos en algunos pacientes, variables diferentes juegan un papel similar (p.ej., evitación del daño y sensación de inacabado) (Rachman, 1994). Finalmente, este trabajo pone de manifiesto la necesidad de seguir trabajando en esta línea para establecer si las diferencias psicopatológicas observadas se corresponden con diferencias en la respuesta al tratamiento, y si, por tanto, es necesario, o al menos recomendable, ajustar los tratamientos a la "motivación" subyacente al lavado.

Nota. Algunas características irrelevantes de los pacientes han sido modificadas. Todos los pacientes dieron su conformidad y cedieron sus datos para la investigación. 


\section{Referencias}

Abramowitz, J. S., Franklin, M. E., Schwartz, S. A., \& Furr, J. M. (2003). Symptom presentation and outcome of cognitive-behavioral therapy for obsessive-compulsive disorder. Journal of Consulting and Clinical Psychology, 71, 1049-57.

American Psychiatric Association. (2000). Diagnostic and statistical manual of mental disorders. Text revised (4th ed.).Washington: American Psychiatric Association.

American Psychiatric Association (2013). Diagnostic and Statistical Manual of Mental Disorders. 5 th edition. Washington, DC: American Psychiatric Association.

Arnáez, S., García-Soriano, G., \& Belloch, A. (2015). Contenidos obsesivos, miedo a la enfermedad y asco. Revista de Psicopatología y Psicología Clínica, 20(1), 33-40.

Belloch, A., Cabedo, E., \& Carrió, C. (2011). TOC. Obsesiones y compulsiones. Tratamiento cognitivo. Madrid: Alianza Editorial.

Belloch, A., Cabedo, E., Carrió, C., Lozano-Quilis, J.A., GilGómez, J.A., \& Gil-Gómez, H. (2014). Virtual reality exposure for OCD. Is it feasible? Revista de Psicopatología y Psicología Clínica, 19(1), 37-44.

Belloch, A., Fornés, G., Carrasco, A., Lopez-Sola, C., Alonso, P., $\&$ Menchon, J. (2016). Incompleteness and not just right experiences in the explanation of obsessive- compulsive disorder. Psychiatry Research, 236, 1-8.

Belloch, A., Morillo, C., Luciano, J. V, García-Soriano, G., Cabedo, E., \& Carrió, C. (2010). Dysfunctional belief domains related to obsessive-compulsive disorder: a further examination of their dimensionality and specificity. The Spanish Journal of Psychology, 13, 376-88.

Carrasco, A., \& Belloch, A. (2013). Algo no está bien: una nueva lectura de la duda obsesiva. Behavioral Psychology / Psicología Conductual, 21, 341-361.

Chik, H. M., Calamari, J. E., Rector, N. A., \& Riemann, B. C. (2010). What do low-dysfunctional beliefs obsessive-compulsive disorder subgroups believe? Journal of Anxiety Disorders, 24, 837-46.

Coles, M. E., Frost, R. O., Heimberg, R. G., \& Rhéaume, J. (2003). "Not just right experiences": perfectionism, obsessive-compulsive features and general psychopathology. Behaviour Research and Therapy, 41, 681-700.

Cougle, J. R., \& Lee, H. J. (2014). Pathological and non-pathological features of obsessive-compulsive disorder: Revisiting basic assumption of cognitive models. Journal of Obsessive-Compulsive and Related Disorders, 3, 12-20.

Cougle, J. R., Goetz, A. R., Fitch, K. E., \& Hawkins, K. A . (2011). Termination of washing compulsions: a problem of internal reference criteria or "not just right" experience? Journal of Anxiety Disorders, 25, 801-805.

Cougle, J. R., Wolitzky-Taylor, K. B., Lee, H.-J., \& Telch, M. J. (2007). Mechanisms of change in ERP treatment of compulsive hand washing: does primary threat make a difference? Behaviour Research and Therapy, 45, 1449-59.

Di Nardo, P.A., Brown, T.A., \& Barlow, D.H. (1994). Anxiety Disorders Interview Schedule for DSM-IV: Life- time version (ADIS-IV-L). New York: Graywind Publications
Ecker, W., \& Gönner, S. (2008). Incompleteness and harm avoidance in OCD symptom dimensions. Behaviour Research and Therapy, 46, 895-904.

Ecker, W., Kupfer, J., \& Gönner, S. (2014). Incompleteness and harm avoidance in OCD, anxiety and depressive disorders, and non-clinical controls. Journal of Obsessive-Compulsive and Related Disorders, 3, 46-51.

Feinstein, S. B., Fallon, B. A., Petkova, E., \& Liebowitz, M. R. (2003). Item-by-Item Factor Analysis of the Yale- Symptom Checklist. Journal of Neuropsychiatry and Clinical Neurosciences, 15, 187-193.

Foa, E. B., Abramowitz, J. S., Franklin, M. E., \& Kozak, M. J. (1999). Feared consequences, fixity of belief, and treatment outcome in patients with obsessive-compulsive disorder. $\mathrm{Be}$ havior Therapy, 30, 717-724.

Fornés-Romero,G., Ruiz, M.A., \& Belloch, A. (2016). Sensación de inacabado y experiencias "no del todo correctas" como motivadoras de los síntomas obsesivo-compulsivos. Revista de Psicopatología y Psicología Clínica, 21, 105-118.

García-Soriano, G., \& Belloch, A. . (2013). Symptom dimensions in obsessive-compulsive disorder: differences in distress, interference, appraisals and neutralizing strategies. Journal of Behavior Therapy and Experimental Psychiatry, 44, 441-448.

García-Soriano, G., Belloch, A., \& Morillo, C. (2008). Sobre la heterogeneidad del trastorno obsesivo-compulsivo. Revista de Psicopatología y Psicología Clínica, 13, 65-84.

García-Soriano, G., Rosell-Clari, V., \& Serrano, M. A. (2016). Emotional and cognitive variables associated with contamination-related obsessive-compulsive symptoms. Spanish Journal of Psychology, 23;19:E25.

Ghisi, M., Chiri, L. R., Marchetti, I., Sanavio, E., \& Sica, C. (2010). In search of specificity: "not just right experiences" and obsessive-compulsive symptoms in non-clinical and clinical Italian individuals. Journal of Anxiety Disorders, 24, 879-86.

Goetz, A. R., Lee, H.-J., Cougle, J. R., \& Turkel, J. E. (2013). Disgust propensity and sensitivity: Differential relationships with obsessive-compulsive symptoms and behavioral approach task performance. Journal of Obsessive-Compulsive and Related Disorders, 2, 412-419.

Goodman, W. K., Price, L. H., Ramussen, S. A., Mazure, C., Delgado, P., Heninger, G. R., \& Charney, D. (1989a). The YaleBrown Obsessive-Compulsive Scale (II): validity. Archives of General Psychiatry, 46, 1012-1016.

Goodman, W. K., Price, L. H., Rasmussen, S. A., Mazure, C., Freischmann, R. L., Hill, C. L., ... Charney, D. S. (1989b). The Yale-Brown Obsessive Compulsive Scale I. Development, Use and Reliability. Archives of General Psychiatry, 46, 1006-1011.

Melli, G., Chiorri, C., Carraresi, C., Stopani, E., \& Bulli, F. (2015). The two dimensions of contamination fear in obsessive-compulsive disorder: Harm avoidance and disgust avoidance. Journal of Obsessive-Compulsive and Related Disorders, 6, 124-131.

Nicolini, H., Herrera, K., Páez, F., Sánchez de Carmona, M., Orozco, B., Lodeiro, G., \& de la Fuente, J. R. (1996). Traducción al español y confiabilidad de la Escala Yale-Brown para el Trastorno Obsesivo-Compulsivo. Salud Mental, 5, 13-16.

Obsessive Compulsive Cognitions Working Group. (2005). Psychometric validation of the obsessive belief questionnaire and 
interpretation of intrusions inventory-Part 2: Factor analyses and testing of a brief version. Behaviour Research and Therapy, 43, 1527-1542.

Olatunji, B. O., Tart, C. D., Ciesielski, B. G., McGrath, P. B., \& Smits, J. A. J. (2011). Specificity of disgust vulnerability in the distinction and treatment of OCD. Journal of Psychiatric Research, 45, 1236-1242.

Rachman, S. (1994). Pollution of the mind. Behaviour Research and Therapy, 32(3), 311-314.

Rasmussen, S.A., \& Eisen, J.L. (1991). Clinical subtypes in OCD. En Y. Zohar, TR Insel, y SA Rasmussen. Psychobiology. New York: Springer-Verlag.

Ruscio, A. M., Stein, D. J., Chiu, W. T., \& Kessler, R. C. (2010). The Epidemiology of obsessive-compulsive disorder in the national comorbidity survey replication. Molecular Psychiatry, 15, 53-63.

Salkovskis, P. M. (1999). Understanding and treating obsessive-compulsive disorder. Behaviour Research and Therapy, 37 Suppl 1, S29-52.

Sandín, B., Chorot, P., Olmedo, M., \& Valiente, R. M. (2008). Escala de propensión y sensibilidad al asco-revisada (DPSS-R): propiedades psicométricas y relación del asco con los miedos y los síntomas obsesivo-compulsivos. Análisis y Modificación de Conducta, 34, 127-168.

Sandín, B., Chorot, P., Santed, M. A., Valiente, R. M., \& Olmedo, M. (2008). Sensibilidad al asco: concepto y relación con los miedos y los trastornos de ansiedad. Revista de Psicopatología y Psicología Clínica, 13, 137-158.

Sandín, B., Chorot, P., Valiente, R. M., \& Santed, M. A. (2014). Dimensiones de sensibilidad al asco y predicción diferencial de los síntomas del trastorno obsesivo-compulsivo. Ansiedad y Estrés, 20, 11-25.

Sandín, B., Valiente, R. M., Chorot, P., Santed, M. A., \& Pineda, D. (2013). Dimensiones de sensibilidad al asco y predicción diferencial de los síntomas fóbicos. Revista de Psicopatología y Psicología Clínica, 18(1), 19-30.

Smith, A. H., Wetterneck, C. T., Hart, J. M., Short, M. B., \& Björgvinsson, T. (2012). Differences in obsessional beliefs and emotion appraisal in obsessive compulsive symptom presentation. Journal of Obsessive-Compulsive and Related Disorders, 1, 54-61.
Sookman, D., Abramowitz, J. S., Calamari, J. E., Wilhelm, S., \& McKay, D. (2005). Subtypes of obsessive-compulsive disorder: Implications for Specialized Cognitive Behavior Therapy. Behavior Therapy, 36, 393-400.

Summerfeldt, L. J. (2004). Understanding and treating incompleteness in obsessive-compulsive disorder. Journal of Clinical Psychology, 60, 1155-68.

Summerfeldt, L. J., Kloosterman, P. H., Antony, M. M., \& Swinson, R. P. (2014). Examining an obsessive-compulsive core dimensions model: Structural validity of harm avoidance and incompleteness. Journal of Obsessive-Compulsive and Related Disorders, 3, 83-94.

Summerfeldt, L. J., Kloosterman, P. H., Antony, M. M., Richter, M. A., \& Swinson, R. P. (2004). The relationship between miscellaneous symptoms and major symptom factors in obsessive-compulsive disorder. Behaviour Research and Therapy, 42, 1453-1467.

Tallis, F. (1996). Compulsive washing in the absence of phobic and illness anxiety. Behaviour Research and Therapy, 34, 361-362.

Tolin, D. F., Brady, R. E., \& Hannan, S. (2008). Obsessional beliefs and symptoms of obsessive-compulsive disorder in a clinical sample. Journal of Psychopathology and Behavioral Assessment, 30, 31-42.

Tolin, D., \& Meunier, S. A. (2007). Contamination and decontamination. En J. Abramowitz, D. Mckay, \& S. Taylor (Eds.), Obsessive-compulsive disorder: subtypes and spectrum conditions (pp. 3-18). Oxford, UK: Elsevier.

Van Overveld, W. J. M., de Jong, P. J., Peters, M. L., Cavanagh, K., \& Davey, G. C. L. (2006). Disgust propensity and disgust sensitivity: Separate constructs that are differentially related to specific fears. Personality and Individual Differences, 41, 1241-1252.

Viar, M. A., Bilsky, S. A., Armstrong, T., \& Olatunji, B. O. (2011). Obsessive beliefs and dimensions of obsessive-compulsive disorder: An Examination of Specific Associations. Cognitive Therapy and Research, 35, 108-117.

Wahl, K., Salkovskis, P. M., \& Cotter, I. (2008). "I wash until it feels right" the phenomenology of stopping criteria in obsessive-compulsive washing. Journal of Anxiety Disorders, 22, $143-61$. 